\title{
Influence de la température et de la vitesse de sollicitation sur les propriétés mécaniques d'alliages ternaires
}

\author{
Stéphane Moreau ${ }^{1,2, a, b}$, Robert Jérisian ${ }^{2, c}$, Thierry Lequeu ${ }^{2}$, \\ Narayanaswami Ranganathan ${ }^{3}$, René Leroy ${ }^{3}$ et Stéphane Méo ${ }^{3}$ \\ 1 Université François-Rabelais de tours, ST Microelectronics, 16 rue Pierre et Marie Curie, \\ BP 7155, 37071 Tours Cedex 2, France \\ 2 Université François-Rabelais de tours, Laboratoire de Microélectronique de Puissance (L.M.P.), ST Microelectronics, \\ 16 rue Pierre et Marie Curie, 37071 Tours Cedex 2, France \\ 3 Université François-Rabelais de tours, Laboratoire de Mécanique et de Rhéologie (L.M.R.), 7 rue Marcel Dassault, \\ 37204 Tours Cedex 3, France
}

Reçu le 16 juillet 2004, accepté le 20 septembre 2007

\begin{abstract}
Résumé - Dans la perspective d'une simulation numérique de type éléments-finis, les auteurs présentent la caractérisation thermomécanique d'alliages ternaires $(92,5 \mathrm{~Pb}-5 \mathrm{Sn}-2,5 \mathrm{Ag}$ et $95,5 \mathrm{~Pb}-2,5 \mathrm{Ag}-2 \mathrm{Sn})$ utilisés dans l'industrie électronique. Au travers d'essais de flexion trois points sur DMA, la présente étude fait ressortir une forte dépendance à la température et à la vitesse de sollicitation du module sécant, de la limite d'élasticité et de la résistance à la rupture. La plage de températures scrutée allant de +35 à $+125^{\circ} \mathrm{C}$ et concernant la vitesse de sollicitation, cette dernière fut choisie entre 0,1 à $10 \mathrm{~N} \cdot \mathrm{min}^{-1}$. Cette forte dépendance à la température des trois grandeurs précitées se caractérise pour le module sécant par une perte de 40 à $70 \%$ par rapport à sa valeur initiale, de 40 à $60 \%$ concernant la limite d'élasticité et de 40 à $45 \%$ pour la résistance à la rupture lorque la température passe de +35 à $+125{ }^{\circ} \mathrm{C}$ et pour une vitesse de sollicitation passant de 0,1 à $10 \mathrm{~N} \cdot \mathrm{min}^{-1}$. Concernant l'influence de la vitesse de sollicitation, on observe que le module sécant grimpe de 35 à $55 \%$, que la limite d'élasticité progresse de 20 à $50 \%$ et que la résistance à la rupture augmente de 15 à $35 \%$ lorsque la vitesse de sollicitation passe de 0,1 à 10 N.min ${ }^{-1}$ et pendant que la température passe de +35 à $+125^{\circ} \mathrm{C}$.
\end{abstract}

Mots clés : Alliage ternaire / 92,5Pb-5Sn-2,5Ag / 95,5Pb-2,5Ag-2Sn / influence de la température / influence de la vitesse de sollicitation / DMA

\begin{abstract}
Effect of temperature and stress rate on mechanical properties of ternary alloys. For FEM-based numerical simulation, one must know the particular mechanical properties. This paper presents a thermomechanical characterization study on two ternary alloys $(92.5 \mathrm{~Pb}-5 \mathrm{Sn}-2.5 \mathrm{Ag}$ and 95.5Pb-2.5Ag-2Sn) used in the electronics industry. By means of Dynamic Mechanical Analysis (DMA), the effects of temperature (from +35 to $+125^{\circ} \mathrm{C}$ ) and stress rates (from 0.1 to $10 \mathrm{~N} \cdot \mathrm{min}^{-1}$ ) on mechanical properties - secant modulus, yield strength and ultimate tensile strength - of $92.5 \mathrm{~Pb}-5 \mathrm{Sn}-2.5 \mathrm{Ag} / 95.5 \mathrm{~Pb}-$ $2.5 \mathrm{Ag}-2 \mathrm{Sn}$ alloy were investigated.
\end{abstract}

Key words: Ternary alloy / 92.5Pb-5Sn-2.5Ag / 95.5Pb-2.5Ag-2Sn / temperature effect / stress rate effect / DMA

\footnotetext{
a Auteur pour correspondance :

stephane.moreau10@orange.fr

b Actuellement, Ingénieur-Chercheur au CEA-LETI, 17 rue

des Martyrs, 38054 Grenoble Cedex 9, France

c Actuellement, Professeur Associé au Centre de Recherches

FORTH de Crète, Grèce
} 


\section{Introduction}

Un TRIAC est un composant électronique permettant le contrôle d'un transfert d'énergie. Ce composant comme de nombreux autres présente deux versions, l'une isolée, l'autre non. Cette isolation s'effectue par l'intermédiaire d'une céramique, en alumine $\left(\mathrm{Al}_{2} \mathrm{O}_{3}\right)$ généralement, brasée entre la connexion d'anode du composant et son radiateur (cf. Fig. 1). Cette isolation se doit d'être effective du point de vue électrique, afin de ne pas retrouver de tension sur le radiateur (danger pour les utilisateurs...), mais pas au regard du transfert énergétique provenant de l'échauffement de la puce de silicium lors du fonctionnement du composant.

De part leur fonction, les composants électroniques tels que les TRIACs sont sujets à de multiples chargements mécaniques dus aux marches/arrêts, aux cycles de fonctionnement... Du fait des différences de Coefficients de Dilatation Thermique (C.D.T.) entre les multiples matériaux constitutifs du composant électronique, les joints de brasures sont fortement soumis aux contraintes et aux déformations causant leur usure et induisent le mauvais fonctionnement du composant électronique à terme.

Lors d'essais de fiabilité de type cycles thermiques (en environnement gazeux, typiquement de l'air) ou chocs thermiques (en environnement liquide), permettant de reproduire de façon accélérée les défaillances rencontrées dans la réalité, les TRIACs de type isolé - c'est-à-dire avec étage céramique - voient leurs joints de brasures au niveau de cette dernière se fissurer (cf. Fig. 2).

Confrontés au raccourcissement de la durée de développement des nouveaux produits, les industriels ont recours de plus en plus souvent aux simulations numériques pour estimer la faisabilité d'un produit, d'une solution technologique... mais également pour déterminer la durée de vie de leur composant sous différentes sollicitations. Cette démarche s'accompagne dorénavant d'un nombre limité d'essais de fiabilité en vue uniquement d'une corrélation. Pour que cette démarche soit efficace, il faut connaître les propriétés mécaniques des matériaux pour remplacer les essais réels par des simulations numériques.

Le maillon faible ${ }^{1}$ vis-à-vis d'une contrainte thermique des TRIACs isolés semblant être les joints de brasures (92,5Pb-5Sn-2,5Ag ou 95,5Pb-2,5Ag-2Sn) au niveau de la céramique [3], les ingénieurs se doivent d'investiguer de manière plus pointue le comportement de ces éléments comparés aux autres éléments qui sont moins sollicités.

Depuis l'avènement de l'électronique, de nombreuses recherches ont porté sur les propriétés mécaniques des alliages de brasures et principalement l'alliage $63 \mathrm{Sn}-37 \mathrm{~Pb}$, commun en assemblage électronique [4-6] mais par contre très peu de résultats concernent les alliages qui nous intéressent.

L'étude actuelle présente les premiers résultats obtenus concernant l'influence de la température et de la

\footnotetext{
${ }^{1}$ Formulation employée en fiabilité électronique, weakestlink en anglais [1,2].
}

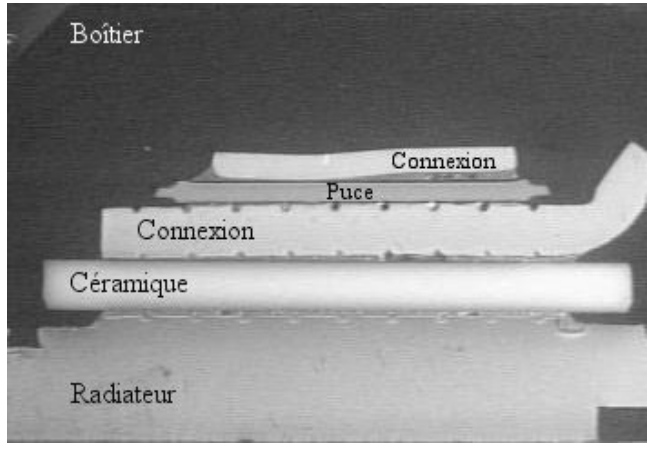

Fig. 1. Coupe transversale d'un TRIAC en version isolée.

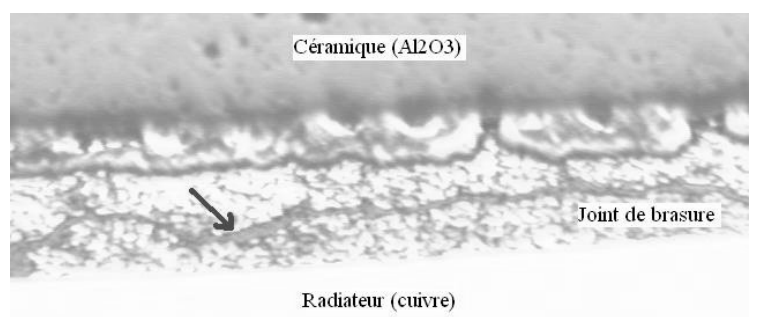

Fig. 2. Joint de brasure fissuré à l'interface cuivre-céramique.

vitesse de sollicitation sur les propriétés mécaniques des alliages ternaires $92,5 \mathrm{~Pb}-5 \mathrm{Sn}-2,5 \mathrm{Ag}$ et $95,5 \mathrm{~Pb}-2,5 \mathrm{Ag}$ 2Sn (module sécant $E_{\mathrm{s}}$, limite d'élasticité $R_{\mathrm{e}}$ et sa résistance à la la rupture $R_{\mathrm{m}}$ ). Pour cette étude les auteurs ont employé un analyseur mécanique dynamique (D.M.A. en anglais pour Dynamic Mechanical Analyzer), mais également les méthodes d'Analyse Thermique Différentielle (A.T.D.) ainsi que d'Analyse ThermoMécanique (A.T.M.) en vue de déterminer les températures de solidification et de fusion ainsi que les coefficients de dilatation thermique de ces alliages.

\section{Procédure expérimentale}

\subsection{Analyse Thermique Différentielle (A.T.D.)}

Le principe de cette méthode consiste à suivre l'évolution de la différence de température $(\Delta T)$ entre l'échantillon étudié et un corps témoin inerte (couramment l'alumine), c'est-à-dire dépourvu d'effets thermiques dans le domaine de température étudié. Cette analyse fournit des informations sur les réactions exo et endothermiques se déroulant au sein du matériau étudié. Les températures de changement de phases, les points de fusions... peuvent être déterminés par cette méthode.

Un A.T.D. de type Perkin-Elmer DTA-7e a été employé durant cette étude [7].

La détermination des points de solidification et de fusion s'est déroulée selon la méthodologie suivante : équilibre à $+50^{\circ} \mathrm{C}$, isotherme de 5 min et début de l'analyse de +50 à $+500{ }^{\circ} \mathrm{C}$ à une vitesse de $5{ }^{\circ} \mathrm{C} \cdot \mathrm{min}^{-1}$. La mesure est effectuée sous atmophère contrôlée (argon) et est répétée quatre fois afin de vérifier la reproductibilité puis une moyenne est ensuite réalisée. Comme pour les 
autres analyses (cf. ci-dessous), chaque essai a été réalisé sur un échantillon différent.

\subsection{Analyse ThermoMécanique (A.T.M.)}

L'Analyse ThermoMécanique (A.T.M.) permet de mesurer les changements dimensionnels en fonction de la température ou du temps. Les mesures peuvent avoir lieu selon différents modes de sollicitation : compression, tension, flexion... Les applications typiques sont la détermination des coefficients de dilatation thermique (C.D.T.), des températures de transition vitreuse...

Un A.T.M. de type Perkin-Elmer TMA-7e a été employé durant cette étude [8].

L'échantillon est placé entre un support en quartz et une pointe dite de pénétration; une force statique de $30 \mathrm{mN}$ est appliquée afin d'être assuré que la pointe est bien en contact avec l'échantillon. La procédure de mesure du coefficient de dilatation thermique est la suivante : équilibre à $-70^{\circ} \mathrm{C}$, isotherme de 5 min et début de l'analyse de -70 à $+270{ }^{\circ} \mathrm{C}$ à une vitesse de $5{ }^{\circ} \mathrm{C} \cdot \mathrm{min}^{-1}$. Le C.D.T. est donné par la pente de la caractéristique 《déplacement-température » ramenée à la hauteur initiale de l'échantillon [8]. La mesure est effectuée sous atmosphère d'hélium et est répétée huit fois puis une moyenne est réalisée.

\subsection{Analyse mécanique dynamique}

L'analyse mécanique dynamique est une technique permettant de mesurer les propriétés mécaniques statiques et dynamiques d'un matériau. Il est ainsi possible de déterminer aussi bien le module d'élasticité, la limite d'élasticité que les lois de fluage, de relaxation et de fatigue.

Dans le présent papier, nous nous limiterons à présenter l'influence de la température et de la vitesse de sollicitation sur les paramètres statiques que sont le module sécant $E_{\mathrm{s}}$, la limite d'élasticité $R_{\mathrm{e}}$ ainsi que la résistance à la rupture $R_{\mathrm{m}}$.

La campagne de tests a été réalisée avec des mors de flexion trois points (cf. Fig. 3) qui permettent de s'affranchir de nombreux problèmes liés notamment à la réalisation des éprouvettes d'essais. Les éprouvettes sont de faibles épaisseurs, pour se rapprocher des épaisseurs des brasures dans les TRIAC et s'affranchir des problèmes de concentrations de contraintes liées aux mors de traction. La forme des mors de flexion limite le glissement de l'éprouvette et les mesures réalisées sur de la céramique nous ont montré que pour ce matériau les résultats sont identiques à ceux que nous avons pu trouver dans la littérature.

Les éprouvettes sont réalisées par la société UMICORE selon le procédé suivant : pesée des différents éléments chimiques puis fonte de ces derniers dans un creuset en graphite avec solidification dans un moule en cuivre (avec un traitement spécifique pour éviter les intermétalliques). Pour les deux alliages, la montée en

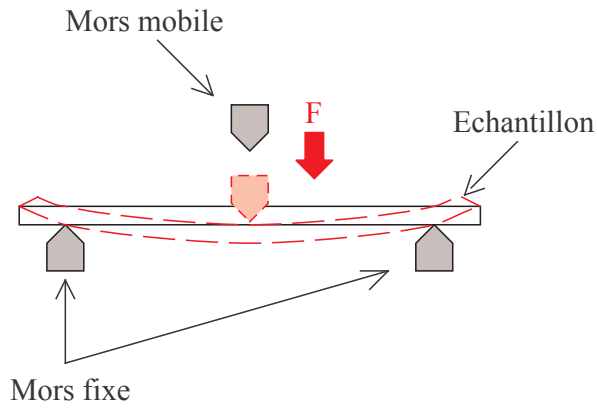

Fig. 3. Principe d'un essai statique en configuration de flexion trois points.

température s'effectue à environ $150^{\circ} \mathrm{C} \cdot \mathrm{min}^{-1}$ et cela jusqu'à $+850^{\circ} \mathrm{C}$. La coulée dans le moule a lieu, après refroidissement à une vitesse d'environ $30^{\circ} \mathrm{C} \cdot \mathrm{min}^{-1}$, à $+450{ }^{\circ} \mathrm{C}$ et tout ceci sous atmosphère d'argon afin d'éviter toute contamination. Les éprouvettes ainsi moulées ont pour dimensions : $200 \times 15 \times 0,5 \mathrm{~mm}$ et sont retaillées par nos soins aux dimensions : $30 \times 15 \times 0,5 \mathrm{~mm}$ en vue d'une utilisation sur un mors de flexion trois points d'entraxe (distance entre les deux points d'appui de l'échantillon) de $15 \mathrm{~mm}$. Cette méthodologie permet de réduire l'impact de la microstructure sur les propriétés des matériaux mesurées puisque les échantillons proviennent de la même coulée.

Les essais sont réalisés en mode « force contrôlée » [9], ainsi au travers de l'expression vitesse de sollicitation, il faut entendre vitesse d'application de la force ou de vitesse de contrainte et non de vitesse de déformation comme dans de nombreux ouvrages issus de la littérature [4-6].

Les mesures ont été réalisées sur un appareil de chez TA-Instruments, le DMA 2980.

La procédure de caractérisation employée est décrite ci-après : pré-charge de $0,05 \mathrm{~N}$, équilibre à la température souhaitée, isotherme de $5 \mathrm{~min}$ et poursuite par une rampe de force, allant jusqu'à la force maximale de la machine à savoir $18 \mathrm{~N}$ et cela à la vitesse de sollicitation désirée. Trois températures et cinq vitesses de sollicitation sont étudiées : $+35 /+70$ et $+125{ }^{\circ} \mathrm{C}$ ainsi que $0,1 / 1 / 4 / 8$ et $10 \mathrm{~N} \cdot \mathrm{min}^{-1}$ (cette dernière étant la limite supérieure de la machine). Chaque test, effectué pour un couple température-vitesse de sollicitation, est répété de deux à quatre fois pour vérifier la reproductibilité et une moyenne est ensuite effectuée.

La contrainte et la déformation prises en compte lors de l'essai de flexion trois points correspondent aux contraintes et déformations maximales intervenant au centre de l'échantillon [10].

\subsection{Récapitulatif des conditions d'essais}

Le tableau 1 rappelle le type de technique employée, les grandeurs mesurées ainsi que les conditions d'essais. 
Tableau 1. Récapitulatifs des conditions d'essais.

\begin{tabular}{|c|c|c|}
\hline Technique & Objectif & Conditions d'essais \\
\hline A.T.D. & $T_{\mathrm{s}}, T_{1}$ & $\begin{array}{l}\text { Équilibre à }+50{ }^{\circ} \mathrm{C} \\
\text { Isotherme : } 5 \text { min } \\
\text { Balayage en température }:+50 \text { à }+500{ }^{\circ} \mathrm{C} \\
\text { Vitesse de balayage }: 5{ }^{\circ} \mathrm{C} \cdot \min ^{-1}\end{array}$ \\
\hline A.T.M. & $\alpha$ & $\begin{array}{l}\text { Équilibre à }+50{ }^{\circ} \mathrm{C} \\
\text { Isotherme }: 5 \mathrm{~min} \\
\text { Balayage en température }:-70 \text { à }+270{ }^{\circ} \mathrm{C} \\
\text { Vitesse de balayage }: 5{ }^{\circ} \mathrm{C} \cdot \mathrm{min}^{-1}\end{array}$ \\
\hline D.M.A. & $E_{\mathrm{s}}, R_{\mathrm{e}}, R_{\mathrm{m}}$ & $\begin{array}{l}\text { Pré-charge : } 0,05 \mathrm{~N} \\
\text { Équilibre à la température souhaitée } \\
\text { Isotherme }: 5 \mathrm{~min} \\
\text { Balayage en force }: 0<F<18 \mathrm{~N} \\
\text { Vitesse de balayage }: 0,1 \text { ou } 1 \text { ou } 4 \text { ou } 8 \text { et } 10 \mathrm{~N} \cdot \mathrm{min}^{-1} \\
\text { Température }:+35 \text { ou }+70 \text { ou }+125^{\circ} \mathrm{C}\end{array}$ \\
\hline
\end{tabular}

\section{Résultats expérimentaux}

\subsection{Températures de solidification et de fusion}

La détermination des températures de solidification $T_{\mathrm{s}}^{2}$ et de fusion $T_{1}^{3}$ s'effectue à partir de la courbe « flux de chaleur-température » (cf. Fig. 4) par la méthode des tangentes [7].

Le tableau 2 présente les valeurs mesurées des températures de solidification et de fusion des deux alliages ternaires et les compare aux valeurs issues de la littérature [11].

Un écart inférieur à $6 \%$ a été noté entre nos mesures et les données issues de la littérature. Cette différence peut s'expliquer par le placement plus ou moins approprié des curseurs servant au calcul des tangentes.

Ces valeurs nous confirment que nous sommes bien en présence d'une brasure tendre puisqu'elle a une température de fusion inférieure à $+450{ }^{\circ} \mathrm{C}$ [12]

\subsection{Coefficient de dilatation thermique (C.D.T.)}

La détermination des C.D.T. s'effectue par la détermination de la pente de la courbe « déplacementtempérature » ramenée à la hauteur initiale de

\footnotetext{
${ }^{2}$ Relatif au solidus : Pour un matériau affecté par un processus de solidification, cristallisation ou fusion, le solidus d'un diagramme de phase sépare le domaine où n'existe que du solide de celui où coexistent solide et liquide. À température croissante, croiser le solidus revient à débuter une fusion partielle; à température décroissante, cela revient à une solidification totale.

3 Relatif au liquidus : à température croissante, croiser le liquidus revient à fondre totalement; à température décroissante, cela revient à débuter une cristallisation partielle.
}

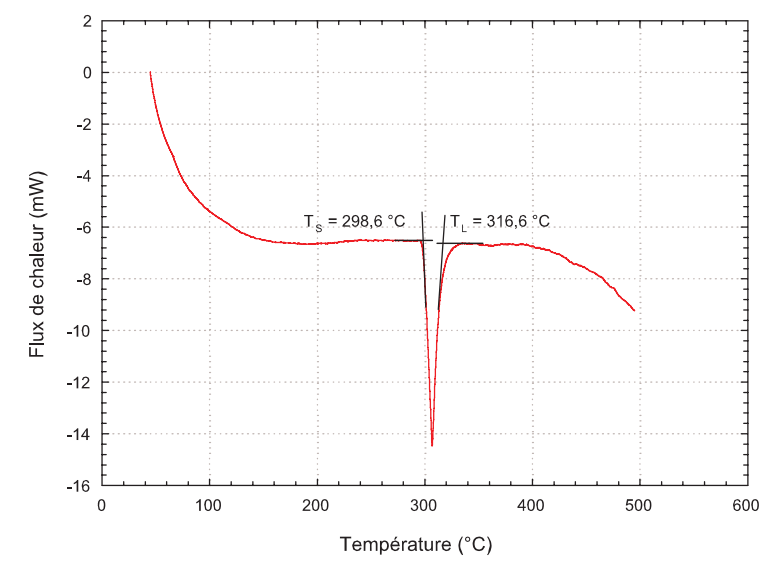

Fig. 4. Évolution du flux de chaleur en fonction de la température et détermination des températures de solidification $T_{\mathrm{s}}$ et de fusion $T_{1}$ par la méthode des tangentes pour l'alliage $92,5 \mathrm{~Pb}-5 \mathrm{Sn}-2,5 \mathrm{Ag}$.

l'échantillon issue d'une mesure de type T.M.A. (cf. Fig. 5).

Le tableau 3 présente les valeurs mesurées des C.D.T. des deux alliages ternaires et les compare aux valeurs issues de la littérature [13].

Nos mesures présentent un écart de l'ordre de $20 \%$ par rapport à la référence [13]. La présence d'impuretés dans les alliages, dans l'un ou l'autre des cas, peut en partie expliquer cet écart.

Le domaine de validité a été limité afin d'éviter que l'alliage ne coule dans le four du T.M.A. mais aussi par rappport à $+150{ }^{\circ} \mathrm{C}$, valeur maximale utilisée lors des simulations. Cette valeur repose elle-même sur la norme MIL-STD-750D méthode 1051.5 ou 1056.7 [14] selon que les essais de fiabilité sont des cycles ou des chocs thermiques. 
Tableau 2. Résumé des températures de solidification et de fusion des alliages ternaires 92,5Pb-5Sn-2,5Ag et 95,5Pb-2,5Ag-2Sn.

\begin{tabular}{lcccc}
\hline Alliage ternaire & \multicolumn{2}{c}{$T_{\mathrm{s}}\left({ }^{\circ} \mathrm{C}\right)$} & \multicolumn{2}{c}{$T_{1}\left({ }^{\circ} \mathrm{C}\right)$} \\
\cline { 2 - 5 } & Mesure & {$[11]$} & Mesure & {$[11]$} \\
\hline $92,5 \mathrm{~Pb}-5 \mathrm{Sn}-2,5 \mathrm{Ag}$ & $298,80( \pm 0,30)$ & 287 & $315,00( \pm 1,00)$ & 296 \\
$95,5 \mathrm{~Pb}-2,5 \mathrm{Ag}-2 \mathrm{Sn}$ & $309,51( \pm 0,16)$ & 299 & $322,20( \pm 0,30)$ & 304 \\
\hline
\end{tabular}

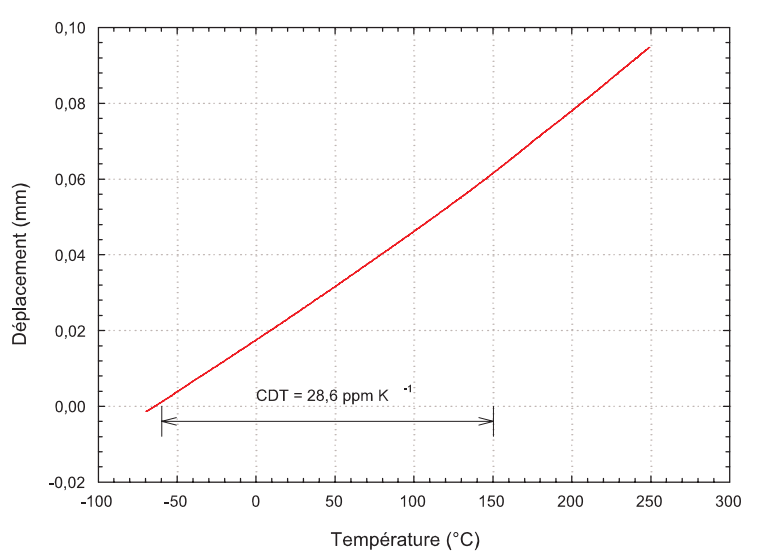

Fig. 5. Évolution de la déformation en fonction de la température et détermination du C.D.T. entre -70 et $+270{ }^{\circ} \mathrm{C}$ pour l'alliage $92,5 \mathrm{~Pb}-5 \mathrm{Sn}-2,5 \mathrm{Ag}$.

Tableau 3. Résumé des coefficients de dilatation thermique des alliages ternaires $92,5 \mathrm{~Pb}-5 \mathrm{Sn}-2,5 \mathrm{Ag}$ et $95,5 \mathrm{~Pb}-2,5 \mathrm{Ag}-2 \mathrm{Sn}$.

\begin{tabular}{lcc}
\hline Alliage ternaire & \multicolumn{2}{c}{ C.D.T. $\left(\times 10^{-6} \mathrm{~K}^{-1}\right)$} \\
\cline { 2 - 3 } & Mesure $\left[-70\right.$ à $\left.+270^{\circ} \mathrm{C}\right]$ & {$[13]$} \\
\hline $92,5 \mathrm{~Pb}-5 \mathrm{Sn}-2,5 \mathrm{Ag}$ & $29,25( \pm 0,76)$ & 24 \\
$95,5 \mathrm{~Pb}-2,5 \mathrm{Ag}-2 \mathrm{Sn}$ & $28,58( \pm 1,20)$ & 23 \\
\hline
\end{tabular}

\subsection{Influence de la température et de la vitesse de sollicitation sur les propriétés mécaniques de l'alliage ternaire}

\subsubsection{Méthodologie d'obtention des paramètres caractéristiques de la courbe contrainte-déformation}

Nous avons calculé la valeur moyenne des deux à quatre essais de reproductibilité de chaque couple température-vitesse de sollicitation.

L'acquisition des données n'étant pas la même d'un essai à l'autre, il nous est impossible de réaliser, sans traiter les données, une moyenne abscisse par abscisse; pour cette raison, nous avons développé un programme sous MATLAB ${ }^{\circledR}$.

Tout d'abord le programme détermine l'essai ayant la déformation la plus faible puis les essais restants sont tronqués par rapport à cette référence. Une interpolation linéaire vis-à-vis de cet essai de référence est effectuée sur les essais tronqués afin d'obtenir des abscisses identiques pour tous les essais. Une fois ce travail effectué une moyenne et un écart-type abscisse par abscisse sont calculés. Ainsi sont obtenus la courbe moyenne des $X$ essais et la dispersion de tous les points de mesure. De cette courbe, sont ensuite extraits les paramètres $E_{\mathrm{s}}, R_{\mathrm{e}_{0,2} \%}$ et $R_{\mathrm{m}}$ qui sont définis dans les sections suivantes.

\subsection{Influence de la température et de la vitesse de sollicitation sur la courbe contrainte-déformation}

En affichant toutes les courbes contrainte--déformation pour chaque vitesse de sollicitation et/ou pour chaque isotherme sur un seul et même graphique, l'effet de la vitesse de sollicitation et/ou de la température sur les propriétés mécaniques de l'alliage ternaire peut être évalué. Les courbes typiques contrainte-déformation à vitesse de sollicitation constante $\left(1 \mathrm{~N} \cdot \mathrm{min}^{-1}\right)$ ainsi qu'à température constante $\left(+35^{\circ} \mathrm{C}\right)$ sont données figures 6 à 9 .

On note facilement que les propriétés mécaniques de ces alliages sont fortement dépendantes des paramètres vitesse de sollicitation et température d'essai. Une augmentation de température a pour effet d'adoucir le matériau. On observe également que le comportement de l'alliage est dépendant de la vitesse de sollicitation; on constate un durcissement du matériau quand cette dernière croît. Ces tendances se retrouvent sur de nombreux alliages contenant ou non du plomb $[15,16]$. L'important de cette étude est de savoir quelles sont les dépendances qui apparaissent vis-à-vis de ces variables sur les paramètres $E_{\mathrm{s}}, R_{\mathrm{e}_{0,2} \%}$ et $R_{\mathrm{m}}$. Les sections suivantes présentent ces dépendances.

Les graphiques présentés dans les sections suivantes sont laissés sans formatage de type semi-log ou log-log car en l'état actuel de l'étude les auteurs préfèrent ne pas tenter de présenter une éventuelle tendance des données. Ce mode de présentation a été choisi d'autant qu'on ne constate pas d'un alliage à l'autre la même dépendance des propriétés mécaniques vis-à-vis de la température et de la vitesse de sollicitation $[4,5,17]$.

\subsubsection{Influence de la température et de la vitesse de sollicitation sur le module sécant}

Contrairement à la céramique ou au cuivre qui composent le TRIAC, les matériaux composant les brasures font que la courbe contrainte-déformation ne présente pas de zone purement élastique, surtout aux températures élevées. Pour pallier ce problème le module sécant $E_{\mathrm{s}}$ est défini [18]. Cette notion est présentée à la figure 10.

Les figures 11 à 14 présentent dans l'ordre, l'influence de la vitesse de sollicitation puis l'influence de la température sur le module sécant pour les deux alliages ternaires. 


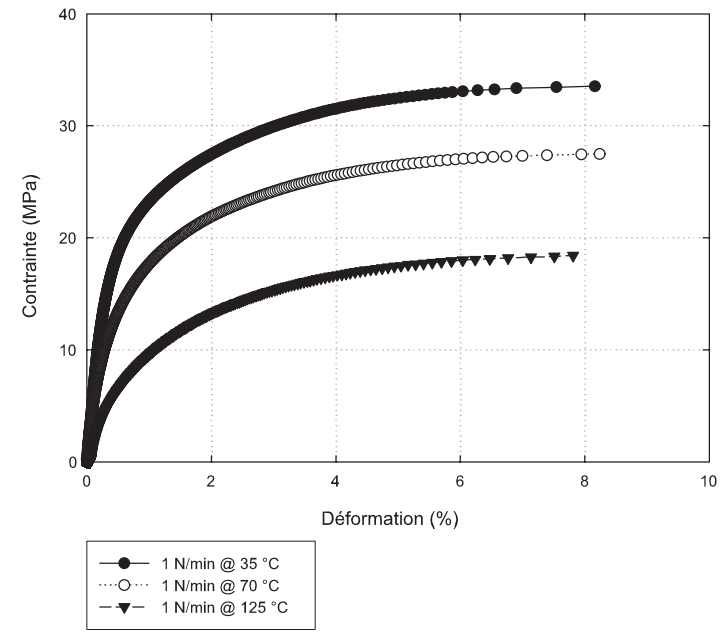

Fig. 6. Influence de la température sur la courbe $\sigma=f(\epsilon)$ pour l'alliage ternaire $92,5 \mathrm{~Pb}-5 \mathrm{Sn}-2,5 \mathrm{Ag}$.

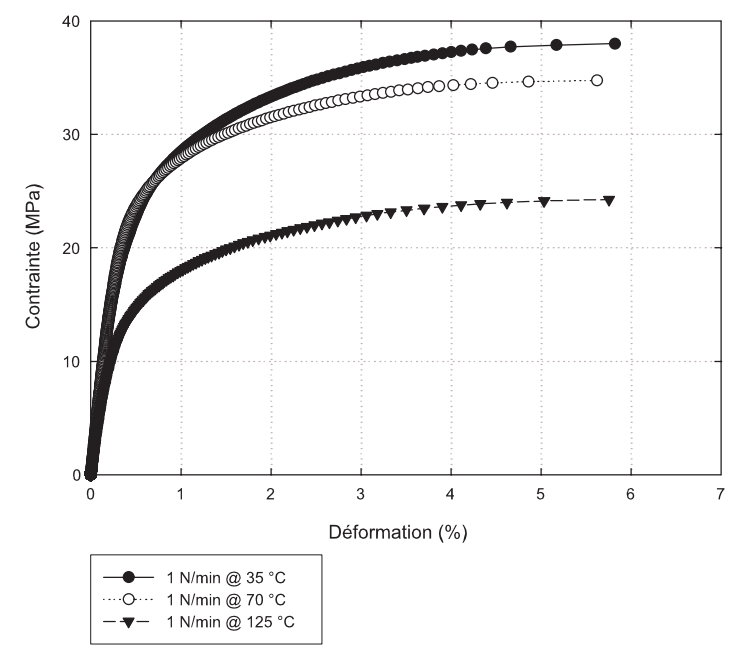

Fig. 7. Influence de la température sur la courbe $\sigma=f(\epsilon)$ pour l'alliage ternaire $95,5 \mathrm{~Pb}-2,5 \mathrm{Ag}$-2Sn.

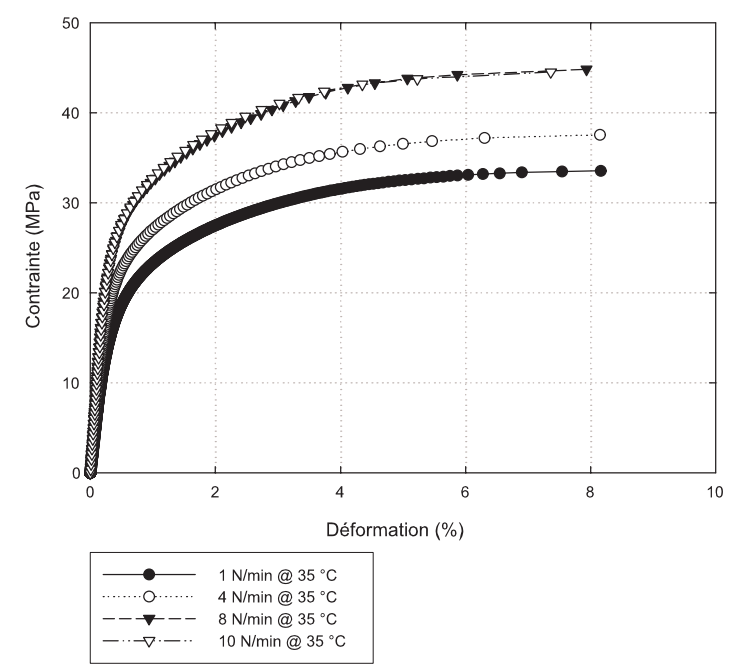

Fig. 8. Influence de la vitesse d'application de la force sur la courbe $\sigma=f(\epsilon)$ pour l'alliage ternaire $92,5 \mathrm{~Pb}-5 \mathrm{Sn}-2,5 \mathrm{Ag}$.

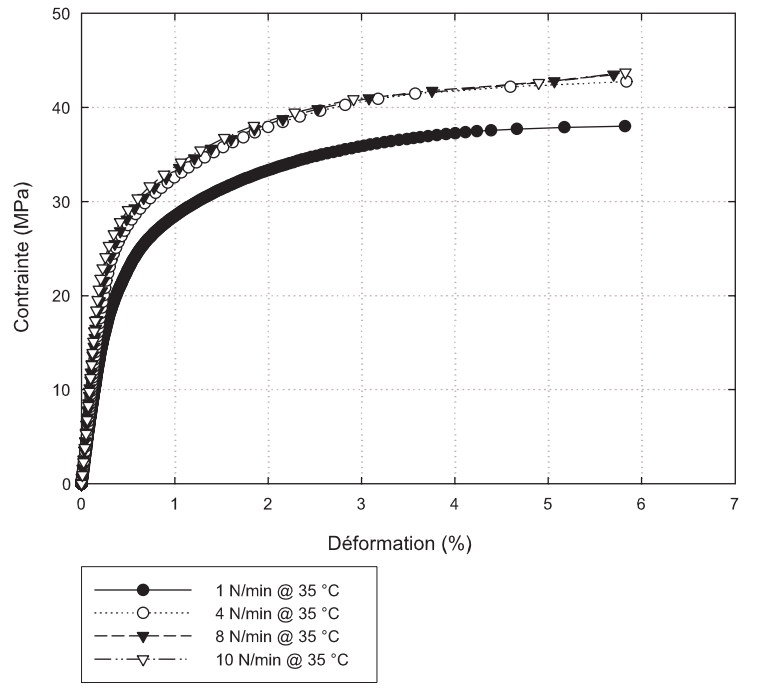

Fig. 9. Influence de la vitesse d'application de la force sur la courbe $\sigma=f(\epsilon)$ sur l'alliage ternaire $95,5 \mathrm{~Pb}-2,5 \mathrm{Ag}-2 \mathrm{Sn}$.

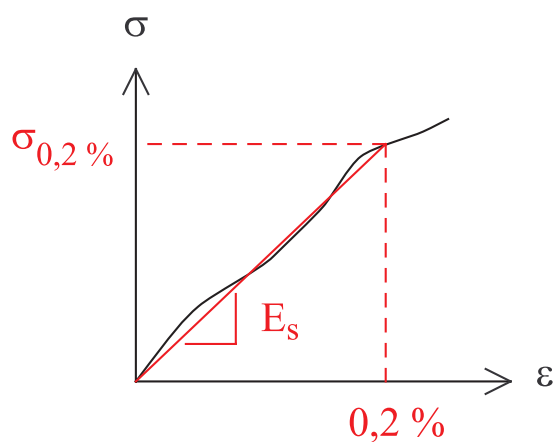

Fig. 10. Rappel concernant la définition du module sécant.

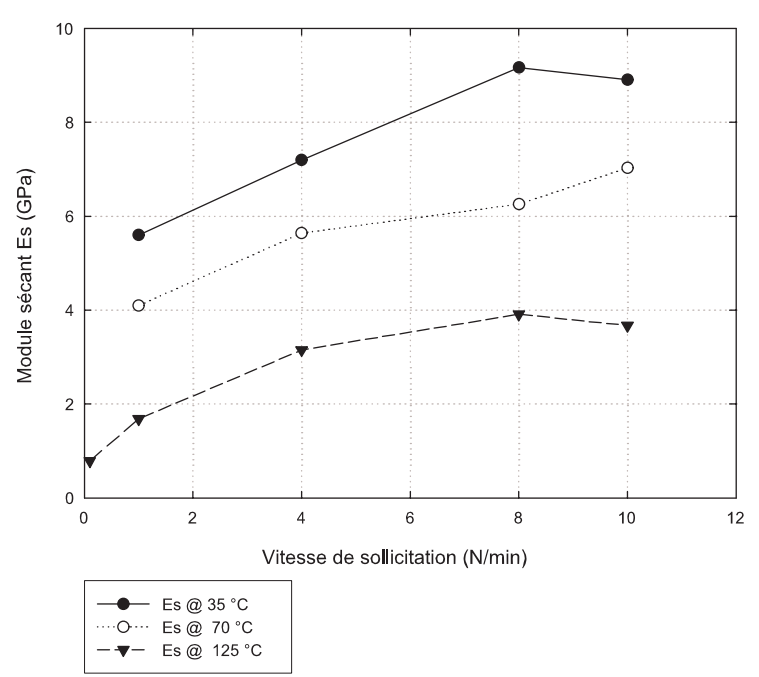

Fig. 11. Influence de la vitesse de sollicitation sur le module sécant pour l'alliage ternaire $92,5 \mathrm{~Pb}-5 \mathrm{Sn}-2,5 \mathrm{Ag}$. 


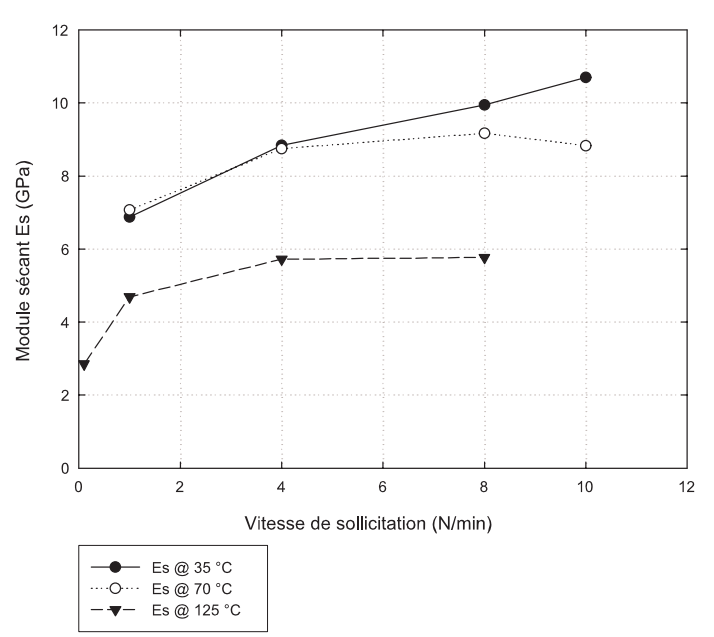

Fig. 12. Influence de la vitesse de sollicitation sur le module sécant pour l'alliage ternaire $95,5 \mathrm{~Pb}-2,5 \mathrm{Ag}-2 \mathrm{Sn}$.

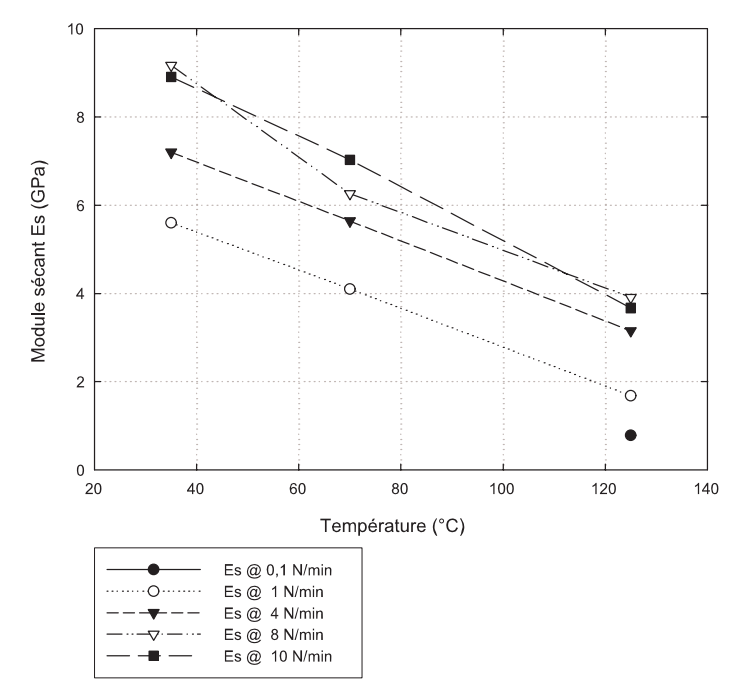

Fig. 13. Influence de la température sur le module sécant pour l'alliage ternaire $92,5 \mathrm{~Pb}-5 \mathrm{Sn}-2,5 \mathrm{Ag}$.

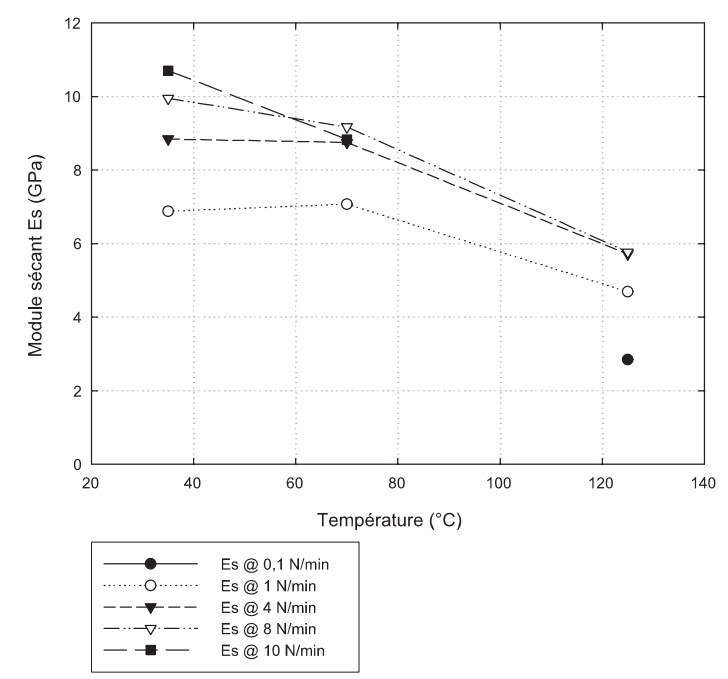

Fig. 14. Influence de la température sur le module sécant pour l'alliage ternaire $95,5 \mathrm{~Pb}-2,5 \mathrm{Ag}-2 \mathrm{Sn}$.
Les deux alliages ternaires présentent un module sécant qui diminue avec la température (de -70 à $-40 \%$ ) et croit avec la vitesse de sollicitation $(\mathrm{de}+35$ à $+55 \%)$.

Le tableau 4 récapitule les données concernant le module sécant pour les deux alliages ternaires et pour tous les couples température-vitesse de sollicitation ainsi que l'écart-type et l'erreur se rapportant à chacun des essais.

\subsubsection{Influence de la température et de la vitesse de sollicitation sur la limite d'élasticité}

Comme pour le calcul du module d'élasticité longitudinal, le calcul de la limite d'élasticité a été déterminé par rapport au module sécant [18]. Par convention, la limite d'élasticité sera définie comme étant la contrainte correspondant à une déformation de $0,2 \%$ et sera notée $R_{\mathrm{e}_{0,2} \%}[19,20]$.

Les figures 15 à 18 présentent l'influence de la vitesse de sollicitation et de la température sur la limite d'élasticité pour chacun des alliages ternaires de l'étude.

Pour les deux alliages ternaires, la limite d'élasticité a tendance à diminuer avec la température (de -60 à -40\%) et à augmenter avec la vitesse de sollicitation $($ de +20 à +50\%).

Le tableau 5 récapitule les données concernant la limite d'élasticité moyenne pour les deux alliages ternaires et pour tous les couples température-vitesse de sollicitation ainsi que l'écart-type et l'erreur se rapportant à chacun des essais.

\subsubsection{Influence de la température et de la vitesse de sollicitation sur la résistance à la rupture}

La valeur correspondant à la contrainte maximum sur la courbe contrainte-déformation est prise comme étant la résistance à la rupture ou résistance mécanique, $R_{\mathrm{m}}$, de l'alliage en cours d'essai. Les figures 19 à 22 montrent l'influence de la vitesse de sollicitation ainsi que de la température sur la résistance à la rupture ou résistance mécanique des alliages ternaires $92,5 \mathrm{~Pb}-5 \mathrm{Sn}-$ $2,5 \mathrm{Ag}$ et $95,5 \mathrm{~Pb} 2,5 \mathrm{Ag} 2 \mathrm{Sn}$.

Les alliages ternaires $92,5 \mathrm{~Pb}-5 \mathrm{Sn}-2,5 \mathrm{Ag}$ et $95,5 \mathrm{~Pb}-$ $2,5 \mathrm{Ag}-2 \mathrm{Sn}$, présentent une résistance à la rupture qui a tendance à diminuer avec la température (de -45 à $-40 \%$ ) et à augmenter avec la vitesse de sollicitation (de +15 à $+35 \%)$.

Le tableau 6 récapitule les données concernant la résistance à la rupture moyenne pour tous les couples température--vitesse de sollicitation ainsi que l'écart-type et l'erreur se rapportant à chacun des essais.

\section{Discussion et perspectives}

L'estimation de la durée de vie d'un composant électronique via une simulation numérique n'est pas chose évidente. Pour obtenir une valeur en phase avec les essais de fiabilité, effectués à titre de corrélation, il est 
Tableau 4. Récapitulatif concernant le module sécant moyen.

\begin{tabular}{|c|c|c|c|c|c|}
\hline Brasure & $\begin{array}{r}T \\
\left({ }^{\circ} \mathrm{C}\right)\end{array}$ & $\begin{array}{r}\mathrm{d} F / \mathrm{d} t \\
\left(\mathrm{~N} \cdot \mathrm{min}^{-1}\right)\end{array}$ & $\begin{array}{r}E_{\mathrm{s}} \text { moyen } \\
(\mathrm{MPa})\end{array}$ & $\begin{array}{r}\text { Écart-type } \\
(\mathrm{MPa})\end{array}$ & $\begin{array}{r}\text { Erreur } \\
(\%)\end{array}$ \\
\hline \multirow[t]{15}{*}{$92,5 \mathrm{~Pb}-5 \mathrm{Sn}-2,5 \mathrm{Ag}$} & +35 & 0,1 & n. a. & & \\
\hline & & 1 & 5603 & 776 & 13,8 \\
\hline & & 4 & 7235 & 1245 & 17,2 \\
\hline & & 8 & 9135 & 763 & 8,4 \\
\hline & & 10 & 8910 & 1971 & 22,1 \\
\hline & +70 & 0,1 & n. a. & & \\
\hline & & 1 & 4095 & 92 & 2,2 \\
\hline & & 4 & 5645 & 247 & 4,4 \\
\hline & & 8 & 6315 & 474 & 7,5 \\
\hline & & 10 & 7040 & 42 & 0,6 \\
\hline & +125 & 0,1 & 787 & 20 & 2,6 \\
\hline & & 1 & 1685 & 177 & 10,5 \\
\hline & & 4 & 3155 & 49 & 1,6 \\
\hline & & 8 & 3900 & 174 & 4,5 \\
\hline & & 10 & 3708 & 328 & 8,8 \\
\hline \multirow[t]{15}{*}{$95,5 \mathrm{~Pb}-2,5 \mathrm{Ag}-2 \mathrm{Sn}$} & +35 & 0,1 & n. a. & & \\
\hline & & 1 & 6885 & 1769 & 25,7 \\
\hline & & 4 & 8825 & 1516 & 17,2 \\
\hline & & 8 & 9965 & 1665 & 16,7 \\
\hline & & 10 & 10845 & 942 & 8,7 \\
\hline & +70 & 0,1 & n. a. & & \\
\hline & & 1 & 7075 & 923 & 13 \\
\hline & & 4 & 8765 & 740 & 8,4 \\
\hline & & 8 & 9222 & 1254 & 13,6 \\
\hline & & 10 & 8885 & 1248 & 14,1 \\
\hline & +125 & 0,1 & 2847 & 114 & 4 \\
\hline & & 1 & 4685 & 732 & 15,6 \\
\hline & & 4 & 5660 & 345 & 6,1 \\
\hline & & 8 & 5762 & 837 & 14,5 \\
\hline & & 10 & n. a. & & \\
\hline
\end{tabular}

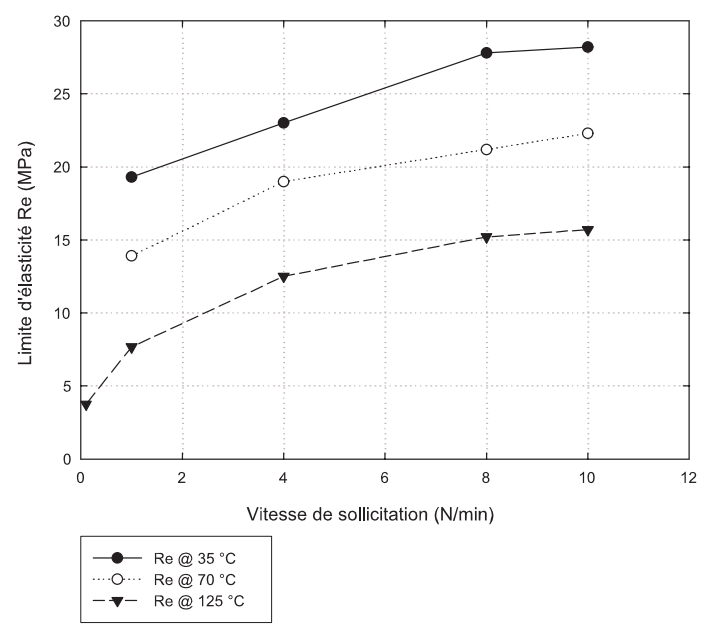

Fig. 15. Influence de la vitesse de sollicitation sur la limite d'élasticité pour l'alliage ternaire $92,5 \mathrm{~Pb}-5 \mathrm{Sn}-2,5 \mathrm{Ag}$.

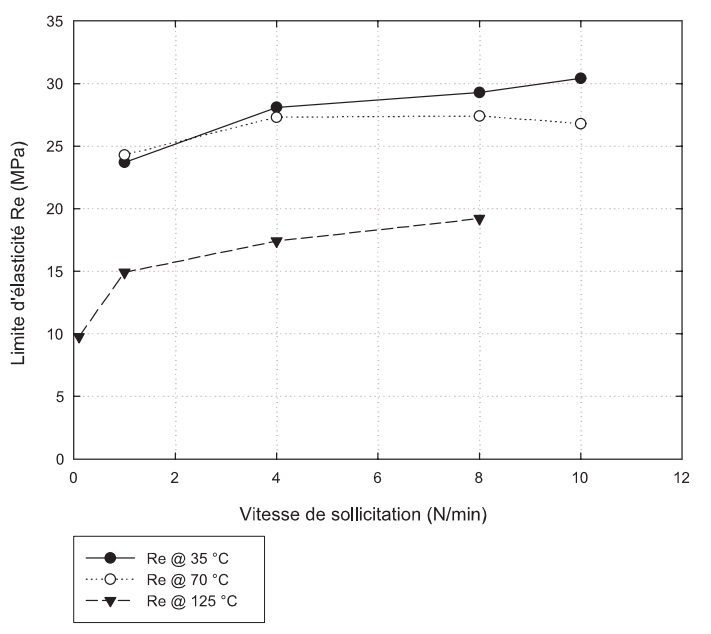

Fig. 16. Influence de la vitesse de sollicitation sur la limite d'élasticité pour l'alliage ternaire $95,5 \mathrm{~Pb}-2,5 \mathrm{Ag}-2 \mathrm{Sn}$. 
Tableau 5. Récapitulatif concernant la limite d'élasticité moyenne.

\begin{tabular}{|c|c|c|c|c|c|}
\hline Brasure & $\begin{array}{r}T \\
\left({ }^{\circ} \mathrm{C}\right) \\
\end{array}$ & $\begin{array}{r}\mathrm{d} F / \mathrm{d} t \\
\left(\mathrm{~N} \cdot \min ^{-1}\right) \\
\end{array}$ & $\begin{array}{r}R_{\mathrm{e}_{0,2} \%} \text { moyenne } \\
(\mathrm{MPa})\end{array}$ & $\begin{array}{r}\text { Écart-type } \\
(\mathrm{MPa}) \\
\end{array}$ & $\begin{array}{r}\text { Erreur } \\
(\%) \\
\end{array}$ \\
\hline \multirow[t]{15}{*}{$92,5 \mathrm{~Pb}-5 \mathrm{Sn}-2,5 \mathrm{Ag}$} & +35 & 0,1 & n. a. & & \\
\hline & & 1 & 19,4 & 2,7 & 14,1 \\
\hline & & 4 & 23,1 & 3,4 & 14,7 \\
\hline & & 8 & 27,8 & 2,2 & 7,8 \\
\hline & & 10 & 28,6 & 2,6 & 9,3 \\
\hline & +70 & 0,1 & n. a. & & \\
\hline & & 1 & 14 & 0,5 & 3,5 \\
\hline & & 4 & 19,1 & 0,1 & 0,7 \\
\hline & & 8 & 21,1 & 0 & 0 \\
\hline & & 10 & 22,4 & 0,1 & 0,3 \\
\hline & +125 & 0,1 & 3,7 & 0,1 & 4 \\
\hline & & 1 & 7,7 & 0,2 & 2,5 \\
\hline & & 4 & 12,4 & 0,1 & 1,1 \\
\hline & & 8 & 15,3 & 0,2 & 1,6 \\
\hline & & 10 & 15,5 & 0,6 & 4,1 \\
\hline \multirow[t]{15}{*}{$95,5 \mathrm{~Pb}-2,5 \mathrm{Ag}-2 \mathrm{Sn}$} & +35 & 0,1 & n. a. & & \\
\hline & & 1 & 24,3 & 1,6 & 6,8 \\
\hline & & 4 & 28,4 & 1,1 & 3,8 \\
\hline & & 8 & 29,1 & 1 & 3,5 \\
\hline & & 10 & 29,8 & 1,2 & 3,9 \\
\hline & +70 & 0,1 & n. a. & & \\
\hline & & 1 & 24,5 & 1 & 4,2 \\
\hline & & 4 & 27,2 & 1,2 & 4,7 \\
\hline & & 8 & 27,4 & 1,4 & 5,2 \\
\hline & & 10 & 27 & 0,6 & 2,3 \\
\hline & +125 & 0,1 & 9,8 & 0,7 & 7,6 \\
\hline & & 1 & 15 & 0,4 & 3,1 \\
\hline & & 4 & 17,4 & 0,7 & 3,8 \\
\hline & & 8 & 19,3 & 1,1 & 5,7 \\
\hline & & 10 & n. a. & & \\
\hline
\end{tabular}

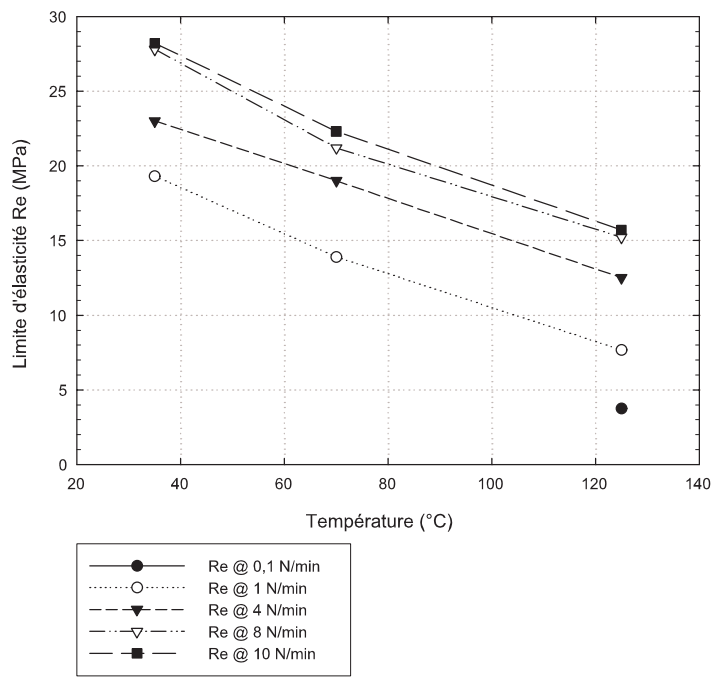

Fig. 17. Influence de la température sur la limite d'élasticité pour l'alliage ternaire $92,5 \mathrm{~Pb}-5 \mathrm{Sn}-2,5 \mathrm{Ag}$.

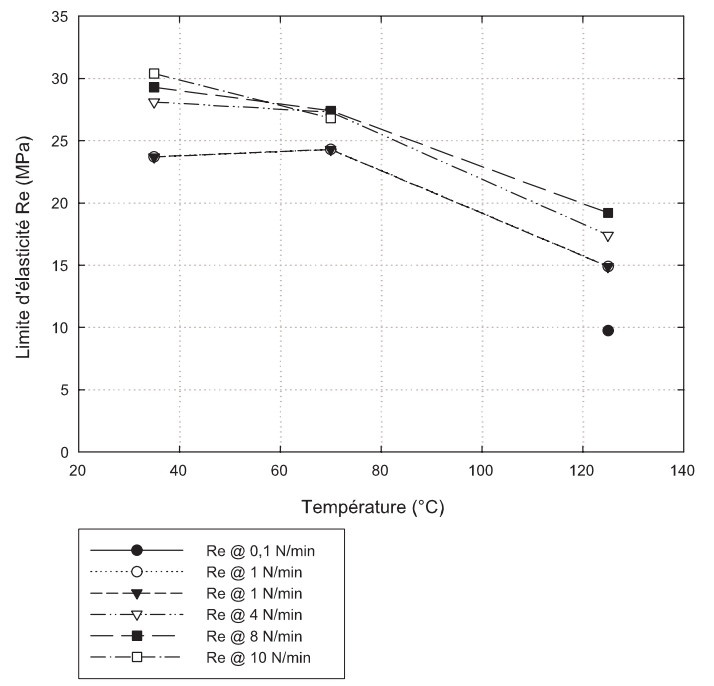

Fig. 18. Influence de la température sur la limite d'élasticité pour l'alliage ternaire $95,5 \mathrm{~Pb}-2,5 \mathrm{Ag}-2 \mathrm{Sn}$. 


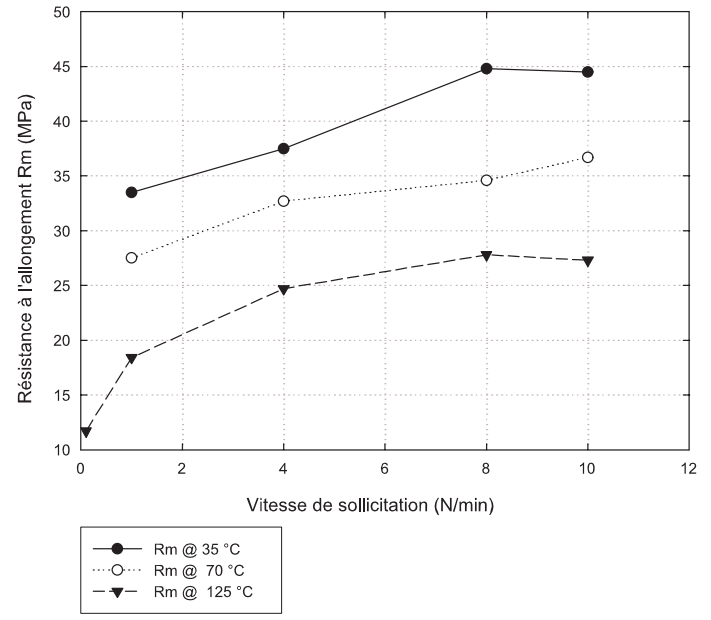

Fig. 19. Influence de la vitesse de sollicitation sur la résistance à la rupture pour l'alliage ternaire $92,5 \mathrm{~Pb}-5 \mathrm{Sn}-$ $2,5 \mathrm{Ag}$.

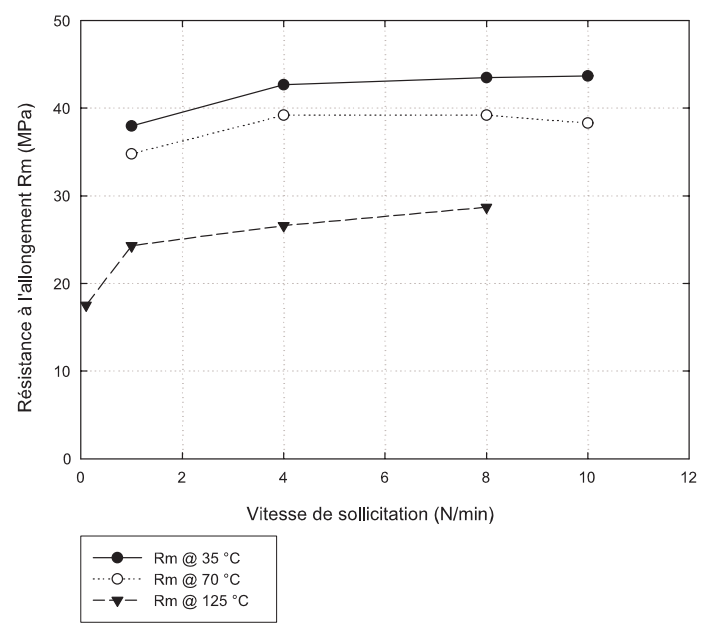

Fig. 20. Influence de la vitesse de sollicitation sur la résistance à la rupture pour l'alliage ternaire $95,5 \mathrm{~Pb}-2,5 \mathrm{Ag}$ 2Sn.

nécessaire de fournir au logiciel de simulation des modèles de matériaux qui correspondent au mieux à la réalité pour un champ d'investigation plus ou moins limité. Les limites sont fixées dans notre cas par les normes définissant les essais de fiabilité de cycles de température et de chocs thermiques [14]. Ainsi les températures maximales admissibles sont de -55 et $+150{ }^{\circ} \mathrm{C}$. Les propriétés des matériaux vont, quant à elles, du simple module de Young, à la loi de fluage en passant par la loi d'écrouissage. L'importance du volume de données à fournir dépend du domaine d'investigation (matériau élastique linéaire ou non, plastique...) et des hypothèses simplificatrices effectuées.

Cette étude s'est uniquement focalisée sur la caratérisation du module sécant, de la limite d'élasticité et de la résistance à la rupture dans une gamme de températures allant de +35 à $+125^{\circ} \mathrm{C}$ et pour des vitesses de sollicitation de 0,1 à $10 \mathrm{~N} \cdot \mathrm{min}^{-1}$.

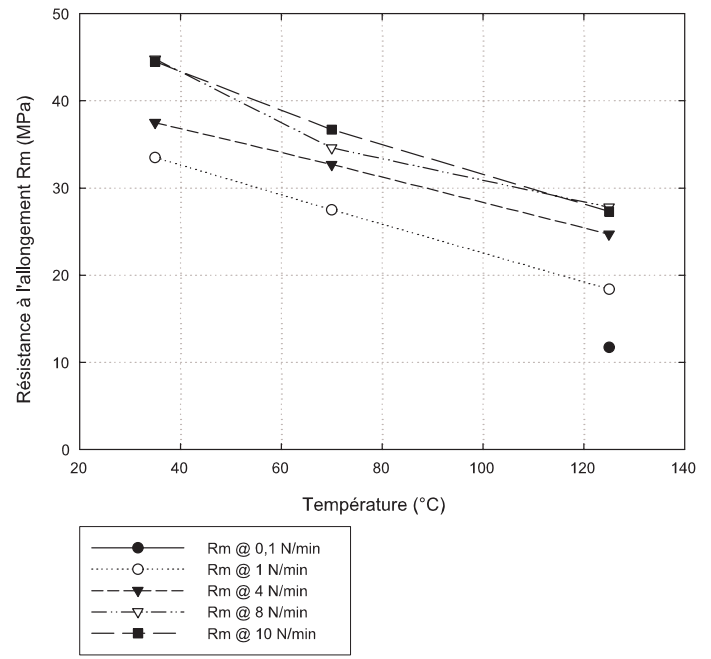

Fig. 21. Influence de la température sur la résistance à la rupture pour l'alliage ternaire $92,5 \mathrm{~Pb}-5 \mathrm{Sn}-2,5 \mathrm{Ag}$.

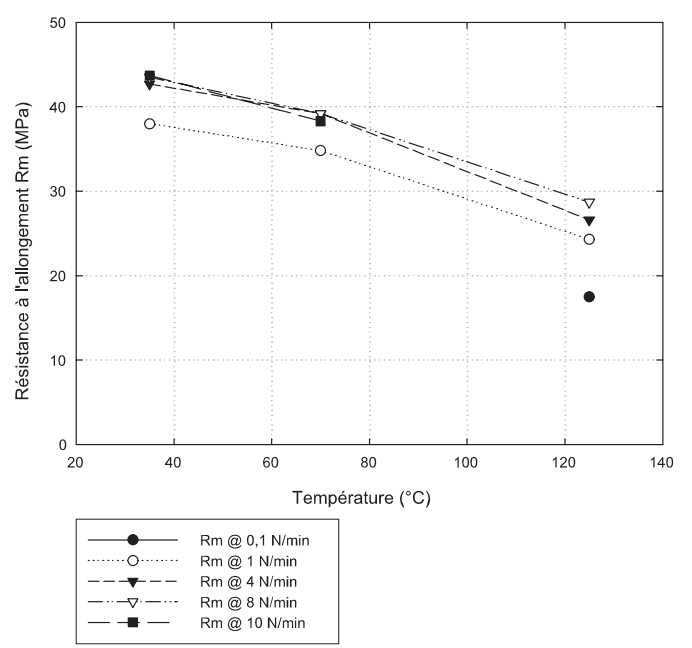

Fig. 22. Influence de la température sur la résistance à la rupture pour l'alliage ternaire $95,5 \mathrm{~Pb}-2,5 \mathrm{Ag}-2 \mathrm{Sn}$.

Au travers de cette étude, nous avons mis en évidence une forte dépendance à la température des trois grandeurs précitées puisqu'en passant de +35 à $+125^{\circ} \mathrm{C}$ et pour une vitesse de sollicitation de $1 \mathrm{~N} \cdot \mathrm{min}^{-1}$, le module sécant perd de 30 à $70 \%$ de sa valeur initiale tandis que la limite d'élasticité diminue de 40 à $60 \%$ et la résistance à la rupture chute de 35 à $45 \%$ (selon l'alliage). De même, on constate une dépendance marquée à la vitesse de sollicitation. Pour une température de $+70{ }^{\circ} \mathrm{C}$ et une vitesse de sollicitation allant de 1 à $10 \mathrm{~N} . \mathrm{min}^{-1}$, on observe que le module sécant croît de 20 à $40 \%$, que la limite d'élasticité progresse de 10 à $40 \%$ et que la résistance à la rupture augmente de 10 à $25 \%$ (selon l'alliage).

Quelle que soit la vitesse de sollicitation, à $+35^{\circ} \mathrm{C}$, l'erreur sur les trois grandeurs est plus élevée que pour les autres températures. Une première explication consisterait à dire qu'au voisinage de cette température, les 
Tableau 6. Récapitulatif concernant la résistance à la rupture moyenne.

\begin{tabular}{|c|c|c|c|c|c|}
\hline Brasure & $\begin{array}{r}T \\
\left({ }^{\circ} \mathrm{C}\right) \\
\end{array}$ & $\begin{array}{r}\mathrm{d} F / \mathrm{d} t \\
\left(\mathrm{~N} \cdot \min ^{-1}\right) \\
\end{array}$ & $\begin{array}{r}R_{\mathrm{m}} \text { moyenne } \\
(\mathrm{MPa})\end{array}$ & $\begin{array}{r}\text { Écart-type } \\
(\mathrm{MPa}) \\
\end{array}$ & $\begin{array}{r}\text { Erreur } \\
(\%) \\
\end{array}$ \\
\hline \multirow[t]{15}{*}{$92,5 \mathrm{~Pb}-5 \mathrm{Sn}-2,5 \mathrm{Ag}$} & +35 & 0,1 & n. a. & & \\
\hline & & 1 & 33,6 & 4,4 & 13,3 \\
\hline & & 4 & 37,6 & 5 & 13,5 \\
\hline & & 8 & 44,9 & 2,1 & 4,6 \\
\hline & & 10 & 44,7 & 3,9 & 8,8 \\
\hline & +70 & 0,1 & n. a. & & \\
\hline & & 1 & 27,5 & 0,8 & 3,1 \\
\hline & & 4 & 32,7 & 0,1 & 0,2 \\
\hline & & 8 & 34,6 & 0,3 & 1 \\
\hline & & 10 & 36,7 & 0,3 & 0,8 \\
\hline & +125 & 0,1 & 11,7 & 0,6 & 5,4 \\
\hline & & 1 & 18,4 & 0,5 & 2,7 \\
\hline & & 4 & 24,7 & 0,2 & 0,9 \\
\hline & & 8 & 27,8 & 0,1 & 0,5 \\
\hline & & 10 & 27,6 & 0,9 & 3,3 \\
\hline \multirow[t]{15}{*}{$95,5 \mathrm{~Pb}-2,5 \mathrm{Ag}-2 \mathrm{Sn}$} & +35 & 0,1 & n. a. & & \\
\hline & & 1 & 38 & 3,5 & 9,2 \\
\hline & & 4 & 42,8 & 1,8 & 4,3 \\
\hline & & 8 & 43,7 & 2,1 & 4,8 \\
\hline & & 10 & 43,7 & 1,5 & 3,4 \\
\hline & +70 & 0,1 & n. a. & & \\
\hline & & 1 & 34,8 & 1,2 & 3,4 \\
\hline & & 4 & 39,4 & 1,6 & 4,2 \\
\hline & & 8 & 39,3 & 1,4 & 3,7 \\
\hline & & 10 & 38,3 & 1,2 & 3,3 \\
\hline & +125 & 0,1 & 17,5 & 1,7 & 10 \\
\hline & & 1 & 24,3 & 0,3 & 1,4 \\
\hline & & 4 & 26,7 & 0,7 & 2,5 \\
\hline & & 8 & 28,8 & 2 & 5,7 \\
\hline & & 10 & n. a. & & \\
\hline
\end{tabular}

défauts de planéité, de positionnement de l'échantillon et par extension de rigidité impliquent une plus grande fluctuation des résultats. Aux températures supérieures ces défauts sont beaucoup plus rapidement atténués. Ainsi, si les erreurs s'étaient limitées à quelques pour-cents, la nécessité d'effectuer un plan d'expériences aurait pu sembler être infondée. Mais la présence d'une erreur d'environ $15 \%$ requiert l'étude de son impact sur le résultat de la simulation numérique.

Concernant les erreurs observées, seul le module sécant semble fortement influencé par les conditions d'essais puisque l'erreur moyenne se situe aux alentours de
$13 \%$ alors que les autres grandeurs présentent une erreur moyenne de $4,5 \%$.

En se focalisant sur l'impact de la composition massique de l'alliage sur les caractéristiques mécaniques, on observe qu'un plus fort pourcentage de plomb permet de diminuer la dépendance à la température et à la vitesse de sollicitation de la caractéristique statique (courbe contrainte-déformation) de l'alliage. Un plus fort pourcentage de plomb permet également d'augmenter les capacités de l'alliage résultant puisque module sécant, limite d'élasticité et résistance à la rupture sont accrus. La saturation à forte vitesse de sollicitation est plus marquée 
pour l'alliage à forte teneur en plomb $(95,5 \mathrm{~Pb}-2,5 \mathrm{Ag}-2 \mathrm{Sn})$ que pour celui de l'étude précédente (92,5Pb-2Sn-2,5Ag).

Malgré le nombre relativement important d'essais réalisés, nous nous sommes rendus compte de la nécessité de poursuivre l'étude notamment dans les températures négatives. La prolongation de cette étude portera sur les températures négatives allant de 0 à $-70{ }^{\circ} \mathrm{C}$ pour les mêmes vitesses de sollicitation que dans les essais présentés ici. Ainsi il sera possible de déterminer les lois d'évolution du module sécant, de la limite d'élasticité et de la résistance à la rupture sur une grande gamme de températures et de vitesses de sollicitation.

Étant donné l'impact conjoint de la température et de la vitesse de sollicitation sur les propriétés mécaniques de ces deux alliages ternaires, il semble très intéressant de caractériser leur comportement en fluage. Cela est d'autant plus vrai que le phénomène de fluage devient non négligeable à partir de $0,5 \times T_{1}$ dans le cas des métaux et alliages métalliques. Or dans la gamme de température étudiée, ces deux alliages ternaires se trouvent dans l'intervalle $0,4 \sim 0,9 \times T_{1}$, par conséquent le fluage de ces alliages ne peut être négligé.

Remerciements. Les auteurs tiennent à remercier M. Hutin de la société UMICORE pour avoir fourni les nombreux échantillons de brasures nécessaires à la présente étude.

\section{Références}

[1] R.B. Abernethy, The New Weibull Handbook, R.B. Abernethy, 4 edition, November 2000

[2] W. Nelson, Applied life data analysis, John Wiley \& Sons Inc, April 1982

[3] S. Moreau, T. Lequeu, R. Jérisian, Comparative study of thermal cycling and thermal shocks tests on electronic components reliability, Microelectron. Reliab. 44 (2004) $1343-1347$

[4] X.Q. Shi, W. Zhou, H.L.J. Pang, Z.P. Wang, Effect of temperature and strain rate on mechanical properties of 63sn/37pb solder alloy, ASME J. Electr. Packaging 121 (1999) 179-185

[5] Li Xiao, J. Liu, Zhonghe Lai, LiLei Ye, A. Tholen, Characterization of mechanical properties of bulk leadfree solders, In Advanced Packaging Materials: Processes,
Properties and Interfaces, Proc. Int. Symp. 6-8 March 2000, pp. 145-151

[6] W.J. Plumbridge, C.R. Gagg, Effects of strain rate and temperature on the stress-strain response of solder alloys, J. Mater. Sci., Mater. Electron. 10 (1999) 461-468

[7] The Perkin Elmer Corporation, Users Manual 7 series/UNIX DTA 7 - Differential Thermal Analyzer, February 1994

[8] The Perkin Elmer Corporation, Users Manual 7 series/UNIX TMA 7 - Thermomechanical Analyzer, February 1994

[9] TA Instruments, Analyse Thermique \& Rhéologie, Analyse Mécanique Dynamique, Manuel d'utilisation, DMA 2980, Vol. 1

[10] TA Instruments, Analyse Thermique \& Rhéologie, Analyse Mécanique Dynamique, Manuel d'utilisation, DMA 2980, Vol. 2

[11] C.A. Harper, Electronic Materials and Processes Handbook, McGraw-Hill, 3 edition, 2004

[12] AFNOR, C90-550, Composants électroniques, Généralités, Alliages, flux et crèmes à braser utilisés pour le brasage tendre, Définition des produits, septembre 1993

[13] http://www.williams-adv.com/packagingMaterials/ lead-free-solder.php, visité le 18 mai 2007

[14] Department of Defense, United States of America, MILSTD-750D, Test method standard, Semiconductor devices, 4th edition, 28 February 1995

[15] P. Chomel, Comportement thermomécanique des alliages métalliques, Techniques de l'ingénieur, Génie mécanique, BD1(BM5012) :BM5012.1-BM5012.36, 2000

[16] L. Anand, Constitutive equations for the rate-dependent deformation of metals at elevated temperatures, ASME J. Eng. Mat. Techn. 104 (1982) 12-17

[17] J.H.L. Pang, B.S. Xiong, C.C. Neo, X.R. Mang, T.H. Low, Bulk solder and solder joint properties for lead free $95.5 \mathrm{sn}-3.8 \mathrm{ag}-0.7 \mathrm{cu}$ solder alloy, In Electronic Components and Technology Conference, Proceedings 53rd, 27-30 May 2003, pp. 673-679

[18] D. François, Essais mécaniques des métaux, Essais de dureté, Techniques de l'ingénieur, Matériaux métalliques, MB2(M123), M123.1-M123.12, 1984

[19] J.P. Baïlon, J.M. Dorlot, Des matériaux, Presses internationales polytechniques, $3^{\mathrm{e}}$ édition, 2000, ISBN 2-55300770-1

[20] J. Lemaître, J.L. Chaboche, Mécanique des matériaux solides, Dunod, Paris, $2^{\mathrm{e}}$ édition, 2001 